\title{
Identificação de pontos de controle no ciclo de desenvolvimento de produto por meio de modelagem conceitual e mapeamento da informação
}

\author{
Identification of control points in the product development cycle \\ through conceptual modeling and information mapping
}

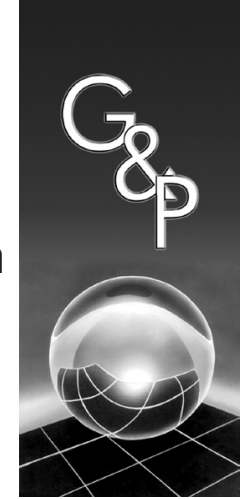

\author{
Leonardo Geraldo de Oliveira Gomes ${ }^{1}$ \\ Andrea Franco Pereira ${ }^{1}$
}

\begin{abstract}
Resumo: A complexidade do sistema produtivo e o conteúdo da informação relacionada a ele são os principais elementos deste trabalho. Traz como fio condutor a tentativa do melhor entendimento desta complexidade e da possibilidade do mapeamento e da representação da informação circulante em um sistema para a otimização de seu gerenciamento. O estudo ganha relevância ao se aproximar das atuais discussões e atitudes emergentes no tocante à sustentabilidade, principalmente nas áreas de desenvolvimento de produtos eco-orientados e no gerenciamento dos ciclos de vida. A proposta fundamenta-se na busca pela forma de representar a complexidade envolvida nos sistemas associados ao desenvolvimento do produto, de maneira a possibilitar a otimização da observação e do controle ao longo do seu ciclo de vida. Esta representação, necessariamente de alto nível, deve apresentar diagramas completos com possibilidades de evolução em detrimento das representações gráficas de baixa complexidade comumente utilizados, caracterizadas pelo reducionismo representativo. Procura-se o controle, o mais fino possível, da cadeia produtiva, objetivando maior organização, produtividade e conhecimento detalhado sobre um produto. O mapeamento da complexidade e a identificação dos pontos de controle propiciam um entendimento otimizado do ciclo de vida. Uma possibilidade de tratar a representação deste ciclo de maneira sistêmica, sem o perigo de uma redução em nível crítico da complexidade, está no uso da modelagem conceitual.
\end{abstract}

Palavras-chave: Complexidade. Representação. Pontos de controle. Modelagem conceitual. Gerenciamento. Produto.

\begin{abstract}
The productive system complexity and its related information content are the main focus of this paper, which seeks an understanding of this complexity and the possibility of mapping and representation of the system information to optimize its management. The present study gains relevance for dealing with sustainability issues, mainly in the development of eco products and lifecycle management. Therefore, the proposal is based on the search for representing the complexity involved in the systems associated with product development in order to optimize the observation and control at the phases in its life cycle. This representation, which is necessarily of high level, should present complete diagrams with scope for improvement instead of the commonly used low complexity graphical representations, characterized by representative reductionism. The focus is on the supply chain control, which should be as tight as possible, aiming at better organization, productivity, and detailed knowledge about a product. Mapping the complexity and the identification of the control points provide a better understanding of the product life cycle. One possibility of having a systematic representation of this cycle, without reducing the critical level of complexity, is through the use of conceptual modeling.
\end{abstract}

Keywords: Complexity. Representation. Control points. Conceptual modeling. Management. Product.

\section{Introdução}

Nos últimos anos, tem-se adquirido consciência de que a maior chance de sobrevivência de nossa sociedade está na autossustentação. Cotidianamente usamos conceitos emergentes como desenvolvimento sustentável, impacto ambiental, ecoeficiência, entre outros. Focando no desenvolvimento de produtos, tem-se procurado formas de desenvolver, obter e utilizar produtos que causem o menor impacto ambiental. Contudo, sabe-se que desenvolver produtos é trabalhar explicitamente de forma interdisciplinar. O processo, ciclo ou cadeia deste desenvolvimento exige conhecimento, observação, participação de áreas de conhecimento distintas. Exige visão sistêmica do conjunto de influências e processamentos. No tocante à sustentabilidade, espera-se o conhecimento e identificação das possibilidades de gestão das

Escola de Arquitetura, Universidade Federal de Minas Gerais - UFMG, CEP 30.130-140, Belo Horizonte, MG, Brasil, e-mail: leolivera-arq@ufmg.br; andreafranco@ufmg.br

Recebido em 1/4/2013 — Aceito em 29/1/2014

Suporte financeiro: Nenhum. 
quantidades de entrada de matéria-prima, saída de refugos e emissões ambientais. Nesse sentido, o alcance de maior ecoeficiência é inerente à abordagem da rastreabilidade já que a ecoeficiência refere-se a uma filosofia de gerenciamento para implantar melhorias ambientais a partir do controle da produção, garantindo ainda ganhos econômicos (PEREIRA; GOMES, 2006). A rastreabilidade é facilitada pela observação e controle dos produtos envolvidos nas etapas do ciclo. Características e comportamentos adquiridos, herdados ou modificados, transformações e ações sofridas propiciam a identificação de pontos de controle para um gerenciamento sistêmico. Pontos de controle são localizações plenas de informação presentes nas linhas de transição de transformações sofridas no fluxo das cadeias de desenvolvimento e produtiva. Contêm informações decisórias que alteram e indicam a direção seguinte no fluxo. São pontos de tradução da interdisciplinaridade característica dos processos de desenvolvimento de produto. A identificação destes pontos auxilia no mapeamento dos locais de contatos entre especializações (e atores) envolvidos. Entretanto, a representação deste mapa exige o respeito a toda a complexidade envolvida e não pode sofrer reducionismo ou possuir representações gráficas de baixa complexidade (comumente utilizadas). É necessário apresentar diagramas completos e incrementais que garantam a evolução da representação do sistema sempre que ele se modificar. Uma possibilidade de mapeamento complexo está no uso da modelagem conceitual. Oriunda das Ciências da Computação é usada para a representação de alto nível do mundo real, sob o ponto de vista de um modelador (ou observador). Os modelos gerados são representativos dos conceitos e características observadas no ambiente e têm total independência das particularidades da implementação (COUGO, 1997). Isto propicia modelação pura e simples de quaisquer sistemas. A informação assume papel fundamental na análise, por tratar-se da representação formal de seus estados assumidos em determinado momento. Compreender o conteúdo informacional existente ao longo do fluxo e relacioná-lo aos atributos dos pontos de controle gera particularidades de gerenciamento que facilitam as tomadas de decisão sem ambiguidades e inteligível às distintas especializações envolvidas.

É evidente a constatação de que a utilização massiva de tecnologia de informação como auxílio ao desenvolvimento e gerenciamento de produtos seja um dos marcos de nosso tempo. Usamos cada vez mais, sistemas digitais integrados, interligados e colaborativos para o gerenciamento de cadeias inteiras e ciclos de vida completos. São os sistemas de CAD (computer aided design), CAM (computer aided manufacturing), CAE (computer aided engineering), PDM (product data management), BIM (building information modeler), LCA (life cycle analysis) e
ERP (enterprise resource planning) que propiciam tais facilidades. Contudo, possuem características e etapas próprias de atuação e exigem um nível de especialização cada vez maior para uma utilização efetiva. As linguagens utilizadas por estes sistemas possuem capacidade de reproduzir nosso mundo real por meio de simulações técnicas e científicas que validam processos de concepção, produção, uso, manutenção e reaproveitamento. Entretanto, a ideia teórica por trás deste estudo foi calcada na possibilidade da utilização de modelagem conceitual para se desenvolver uma metodologia de representação da complexidade para aplicação em uma etapa anterior de uso dos citados sistemas (antes do início da fabricação ou obra).

\subsection{Abordagens sobre sistemas e complexidade}

As teorias e linhas de pensamento sobre sistemas abordadas nesta análise têm como característica a relação com o sistema produtivo. $\mathrm{O}$ consenso da definição de sistemas indica um conjunto de elementos interconectados, formando um todo organizado, com um objetivo geral a ser atingido. É modo de organização, meios, processos, métodos e formas. A integração entre os componentes se dá por fluxo de matéria e energia, comandados e/ou controlados por fluxo de informações. Sua complexidade é relativa ao número de atores envolvidos, interligações e processos ocorridos, nos quais os resultados modificam o ambiente em que atua o sistema. Somam-se conceitos relacionados aos eventos ocorridos ao longo do tempo, do controle, reação e comunicação das máquinas (mecânicas, digitais ou biológicas) e às mudanças decorrentes disso (BERTALANFFY, 2008; WIENER 1965). O observador e o ator ganham relevância e ampliam como agentes da estabilização de um sistema. Um sistema também é definido por sua fronteira e o ambiente envolvente, sendo que dentro deste está a zona de menor complexidade e no seu entorno a de maior complexidade. A comunicação opera entre as zonas processando informações necessárias para manter a estabilidade do sistema (LUHMANN apud AMARO, 2010).

Com o objetivo de modelização dessa complexidade, define-se que sistema é uma rede limitada de processadores elementares visando uma intervenção finalizante sobre a envolvente. A estrutura dos elementos, as relações entre eles e o conjunto das atividades que se desenvolvem num tempo são referenciais (LE MOIGNE, 1995). Eles podem ser classificados em duas categorias básicas: os sistemas conceituais e os físicos. Um sistema conceitual é composto de ideias ou teorias e só existe pela consciência, observação ou em virtude do sistema conceitual em si. É responsável pela comunicação 
e compreensão do todo (PLOTKIN, 2003). Um sistema físico é composto de matéria e energia e pode ser observado ou experimentado. Sua principal característica é conter ou estar contido em outros sistemas físicos que, por sua vez, são compostos por objetos (PLOTKIN, 2003).

\subsection{O sistema produtivo e sua complexidade}

Sistema produtivo é o encadeamento de atividades econômicas, nas quais passam e são transformados e transferidos insumos (matérias-primas, máquinas e equipamentos, produtos intermediários, distribuição etc.). Resulta e implica divisão de trabalho, na qual cada agente especializa-se em etapas distintas do processo produtivo (LASTRES; CASSIOLATO, 2003). Tem-se uma cadeia produtiva ao se acrescentar o conceito geográfico. Os Arranjos Produtivos Locais (APL) envolvem a própria cadeia produtiva, a distribuição e comercialização de bens e serviços e as interações com outros atores intervenientes como governo, instituições de crédito, ensino e pesquisa (PEREIRA; GOMES, 2006).

A complexidade está no lidar com a enorme rede de atores, instituições e organismos, todos se relacionando direta e/ou indiretamente, na qual uma multiplicidade de fatores, ações, processos, decisões e responsabilidades estão presentes. Trata-se, na verdade, de vários sistemas complexos relacionando entre si, necessitando de contatos, de interligações e de traduções para as soluções próprias e autorreguladoras que adotam para existir (LUHMAN apud AMARO, 2010). E, para que tudo isso seja ao mesmo tempo complexo e estável, é necessário que associe de maneira coordenada um número limitado de interações a uma grande quantidade de elementos (PEREIRA; GOMES, 2006). A partir do conhecimento destas interações (elementos e atores envolvidos), é possível "conhecer" o objeto a se desenvolver ou produzir, interpretando-o a fim de antecipar comportamentos em situações variadas (LE MOIGNE, 1995). A complexidade também está relacionada com a informação potencial contida no sistema e é importante perceber que ela assume diversos formatos, se materializando ao longo do tempo de desenvolvimento ou produção. Esta materialização da informação ocorre sob vários tipos de suportes (documentos, objetos, protótipos etc.). Contudo, apesar da diversidade, o conteúdo informacional destes suportes representa um mesmo produto.

\subsection{Metodologias de gerenciamento de sistemas}

A etapa de desenvolvimento do produto vai desde a identificação de oportunidades até o lançamento do produto, passando pela pesquisa de mercado, seleção de conceito, projeto de produto e processos de pré-produção (CHENG; LEONEL FILHO, 2006). A validação dos processos de pré-produção propicia uma produção caracterizada pela repetibilidade. Obter sucesso no desenvolvimento de um produto significa saber integrar agentes externos (parcerias, fornecedores e clientes) e internos (áreas funcionais desde a pesquisa até a produção). Metodologias de GDP (Gerenciamento de Desenvolvimento de Produto), tais como Design for Six Sigma, Engineering Methods for Robust Product Design, Engenharia Simultânea, Technology Roadmapping, STAGEGATE Development New Products ou PDMA fornecem pontos conceituais que interessam à gestão de complexidade (CHENG; LEONEL FILHO, 2006; CNH..., 2000; SILVEIRA, 2006; MAXIMINIANO, 2002). Analisar metodologias de gestão propicia conhecimento de procedimentos já validados. Elas apresentam pontos de convergência de conceitos no tocante aos processos, atores e comportamentos. Os mais pertinentes definem que: 1) processos devem ser divididos em estágios com controles intermediários; 2) pontos de decisão são mecanismos de controle de qualidade e critérios para continuidade de fases; 3) processos de pré-desenvolvimento são decisórios; 4) são necessárias equipes especializadas interfuncionais; 5) requerem mudanças na cultura organizacional; 6) é necessário o gerenciamento de riscos; 7) existem processos paralelos de equilíbrio; e 8) recomendam a adoção das boas práticas de gestão (CHENG; LEONEL FILHO, 2006; CNH..., 2000; SILVEIRA, 2006; MAXIMINIANO, 2002).

\section{Abordagens sobre a modelagem conceitual}

A modelagem conceitual é entendida como o ponto de partida para os processos de modelagem lógica de dados, que é parte fundamental no desenho de um sistema de informação, definindo a especificação das estruturas de dados e seus relacionamentos. Em um modelo conceitual, os objetos têm sua representação fiel ao ambiente observado (COUGO, 1997). O uso do modelo conceitual é indicado para a fase de conversação, entendimento, transmissão, validação de conceitos e mapeamento do ambiente. É ferramenta da fase de análise e conceituação e não da fase de execução (GOMES, 2011).

\subsection{Os objetos}

Um objeto é reconhecido no mundo por suas características, funções e forma de se relacionar com atores ou com outros objetos. Os objetos do mundo real compartilham duas características principais: estado e comportamento. Além destas características, os objetos carregam atributos que os definem e os classificam. 
Quando se observam objetos em um ambiente, se está, na verdade, classificando-os por seus atributos. Por meio da análise dos atributos, pode-se enquadrar os objetos numa entidade chamada Classe, que agrega objetos por atributos caracterizadores identificados pelas finalidades: 1) descritiva, que representam atributos intrínsecos ao objeto; 2) nominativa, que se aplica à definição de nome ou rótulo de identificação; e 3) referencial, que se aplica à associação de um objeto com uma alocação (COUGO, 1997). O trabalho de modelagem com o foco no objeto dentro de um limite observável de mundo tem na Modelagem Orientada a Objeto sua base teórica e conceitual.

\subsection{A Modelagem Orientada a Objetos (MOO)}

A MOO é um paradigma de desenvolvimento de sistemas de informação que possui fortes bases conceituais e se apresenta como um dos melhores métodos de abstração de conceitos do mundo real. Por sua qualidade na geração semântica de modelos conceituais, é base de trabalho para o campo da inteligência artificial e da linguística. A MOO tem como meta identificar o melhor conjunto de objetos para descrever um sistema, cujo funcionamento se dá por meio do relacionamento e troca de mensagens entre os objetos (MELO, 2004; WILSON FILHO, 2000). Ela acrescenta, na modelagem conceitual, conceitos que otimizam a modelação da complexidade dos sistemas, como os: 1) Métodos - que definem as habilidades dos objetos; 2) Herança - que permite que uma classe possa se estender a outra aproveitando seus atributos e métodos; 3) Encapsulamento - que consiste na separação de aspectos internos e externos de um objeto; e 4) Abstração - que é a habilidade de se concentrar nos aspectos essenciais de um contexto, ignorando características menos importantes ou acidentais (MELO, 2004). Uma programação baseada na orientação a objetos implementa um conjunto de classes que definem os objetos de um sistema, determinando seus estados (atributos), comportamentos (métodos) e relacionamentos, tornando mais rica em semântica a modelagem conceitual (significação).

\subsection{As ferramentas de UML (Unified Modeling Language)}

A UML é uma linguagem padronizada para a modelagem de sistemas de software orientados a objetos que pode ser trabalhada de forma conceitual (somente modelo) ou para integração com banco de dados e ferramentas CASE (Computer-Aided Software Engineering) (ERIKSSON et al., 2004). Trata-se de ferramenta de modelagem e de auxílio na visualização do desenho de um sistema, sendo baseada em diagramas padronizados que possuem notação gráfica e que também especificam significados, isto é, semântica. É baseada na compilação das "melhores práticas" de Engenharia de Software. Seus diagramas preveem quase toda possibilidade de ações, processos, arranjos, interações, tarefas etc. (ERIKSSON et al., 2004; CARDOSO, 2003). Um modelo de um sistema "desenhado" com UML descreve como ele será, mas não diz como será implementado.

\section{Abstrações sobre sistemas, gestão e modelagem conceitual}

Utilizando o conceito de abstração da MOO, pode-se isolar atributos dos objetos de forma a se identificar tópicos conceituais para definição de possíveis pontos de controle. Tais pontos atuam nos momentos de se decidir sobre continuar, retornar ou abortar um desenvolvimento ou produção. É quando são avaliados os critérios (pré-definidos ou não) que devem ser atendidos antes que as entregas (resultados dos processos) derivadas das etapas anteriores sejam processadas e deem continuidade ao fluxo (atuam onde existe a possibilidade de uma alteração no fluxo do ciclo). O desenvolvimento de produto envolve a solução de problemas de concepção ou produção, por um ator ou grupo deles, baseados em referências (ou parâmetros) que são partes integrantes de todos os processos organizacionais ou produtivos.

A Tabela 1 mostra como poderia ser a abstração sobre os relacionamentos entre os conceitos de ator, operário e observador, baseando-se nos seus atributos estabelecidos e descritos por alguns autores relacionados (BERTALANFFY, 2008; LE

Tabela 1. Exemplo de abstração de conceitos.

\begin{tabular}{llll}
\hline \multicolumn{1}{c}{ Objeto } & \multicolumn{1}{c}{ Função } & \multicolumn{1}{c}{ Ação } & \multicolumn{1}{c}{ Atuação } \\
\hline ATOR & $\begin{array}{l}\text { Propor processos e decidir } \\
\text { por eles }\end{array}$ & $\begin{array}{l}\text { Analisar a orientação seguinte do } \\
\text { fluxo dos processos do sistema }\end{array}$ & $\begin{array}{l}\text { No sistema, no seu entorno. } \\
\text { OPERÁRIO }\end{array}$ \\
$\begin{array}{llll}\text { Procurar por uma } \\
\text { estabilidade no sistema }\end{array}$ & $\begin{array}{l}\text { Realizar processos que buscam } \\
\text { a validação dos objetivos do } \\
\text { sistema }\end{array}$ & No sistema e nos objetos. \\
\hline OBSERVADOR & $\begin{array}{l}\text { Identificar e especificar } \\
\text { estruturas de dados e seus } \\
\text { relacionamentos }\end{array}$ & $\begin{array}{l}\text { Representar os conceitos e } \\
\text { atributos de um objeto num dado } \\
\text { ambiente }\end{array}$ & $\begin{array}{l}\text { No sistema, no seu entorno, nos } \\
\text { eventos de transição sistema/ } \\
\text { entorno, em um objeto e nos } \\
\text { eventos de transição sistema/objeto. }\end{array}$ \\
\hline
\end{tabular}

Fonte: Elaborado pelos autores. 
MOIGNE, 1995; CHENG; LEONEL FILHO, 2006; COUGO, 1997; CARDOSO, 2003). Isto possibilita um mapeamento das ações e dos limites de atuação daqueles conceitos; o que é útil aos processos de gestão. Pode-se então, identificar e mapear possíveis locais de controle (ou parâmetros) a partir das abstrações propostas, por exemplo, para o critério ATUAÇÃO da Tabela 1 (Figura 1):

Pela definição formal, parâmetro é todo elemento cuja variação de valor altera a solução de um problema sem, contudo, alterar sua natureza. Também define como uma variável de um sistema deve se comportar. Atribuem-se valores aos parâmetros para se alterar ou validar comportamentos (MEADOWS, 1995). Caso se identifique grande quantidade de parâmetros é possível organizá-los em uma classe de parâmetros (Figura 2).

O mapeamento na Figura 2 mostra o conteúdo de seis classes de parâmetros abstraídas dos conceitos sobre sistemas, complexidade, gerenciamento e modelagem conceitual, abordados anteriormente e que são pertinentes a este estudo. Na prática, os resultados dos processos de desenvolvimento de produto, como os escopos de projetos, os conceitos de produtos ou os arranjos de procedimentos, devem ser analisados e abstraídos à exaustão com o objetivo de identificação de possíveis locais de controle, de pontos de controle e parâmetros. Deverão ser identificados quantos pontos de controle forem necessários a uma gestão eficiente e todos devem ser representados por mapeamentos.

A Figura 3 apresenta o detalhe da classe Objeto seguida de um exemplo de como se procede a uma análise de identificação de parâmetros de controle.

Deve-se prever, no mínimo, um ponto de controle para: 1) Objeto: a identificação dos atributos (que definem o objeto); 2) Codificação: que personifica o objeto em função de identificações possíveis dele e de seus arranjos (GOMES, 2008); 3) Observação: para a definição das relações do objeto e seu entorno (limites objeto-mundo); e 4) Resolução: para a definição do grau de detalhamento necessário para controle (observação mais "detalhada" do objeto ou mais "detalhada" do sistema a que ele pertence).

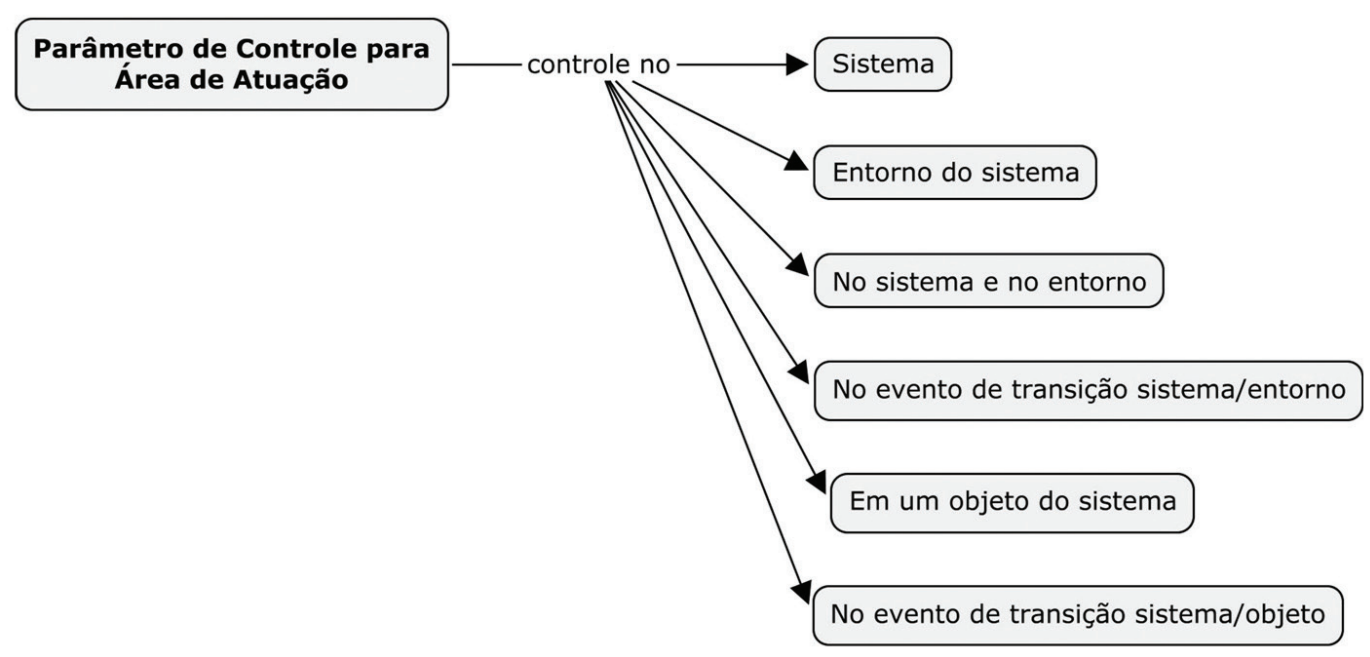

Figura 1. Exemplo de parametrização dos locais de atuação dos sistemas. Fonte: Elaborado pelos autores.

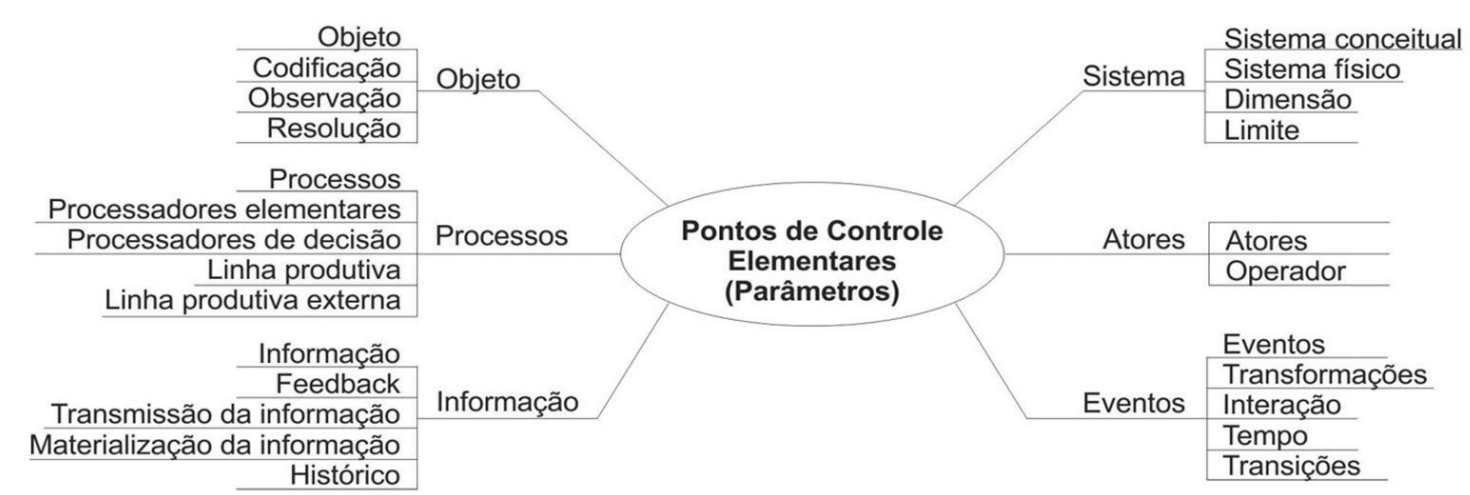

Figura 2. Mapeamento das classes de parâmetros. Fonte: Elaborado pelos autores. 


\section{A representação da complexidade e dos pontos de controle}

Um diagrama de representação para o gerenciamento do ciclo de vida deve ser capaz de representar a complexidade envolvida sem reducionismo, além de poder ser incrementado sempre que novos atores, processos e eventos se mostrarem necessários. Nele deve-se identificar as linhas de relacionamentos e de feedback da informação, os fluxos de emissões na linha produtiva e a dimensão do sistema modelado, inclusive com as linhas de relacionamentos dos atores intervenientes. Os pontos de controle são identificados nas linhas de transição entre os estados de transformações na linha produtiva. É evidente a importância do modelador (observador) neste processo. Ele deve dispor da capacidade de conceber uma multiplicidade de pontos de vista, incluindo o seu próprio, pois a modelação consiste em sua capacidade de identificar características e locais em relações, nos quais se possam interpretar e representar os fenômenos que geram a ação e, consequentemente, os eventos.

É prender-se na complexidade (MORIN; LE MOIGNE, 2000). Isto significa reconhecer que a modelização se constrói como um ponto de vista sobre o real, no qual um trabalho de ordenamento, parcial e continuamente adaptável, pode ser posto em prática (COUGO, 1997).

Para se conseguir modelar e se obter mapas de representação da complexidade do sistema produtivo, foram desenvolvidos dois diagramas (Diagrama de Relação Sistema-Sistema e Diagrama Objeto-transformação) e utilizados outros dois diagramas que são disponibilizados pela UML (Diagrama de caso de Uso e Diagrama de Atividades). A ferramenta UML trabalha a modelação com 12 diagramas.

\subsection{O diagrama para as relações Sistema-Sistema}

É um modelo de diagrama (Figura 4) que cuida do mapeamento das relações entre vários sistemas, do seu entorno e de todos os eventos das transições entre eles (sistema conceitual $\mathrm{x}$ sistema físico, sistema físico $\mathrm{x}$ entorno e vice-versa). Para esta fase da modelagem, devem ser observadas e delimitadas todas as possíveis transições entre sistemas e objetos. O modelador precisa perceber o momento correto de "sair" de um sistema para observá-lo como objeto ou "entrar" em um objeto para observá-lo como sistema. Este processo afeta a resolução de observação do sistema (o grau de detalhamento necessário para o controle efetivo), consequentemente afetando os limites, as dimensões e a complexidade das relações que serão modeladas. A ampliação dos limites e dimensões, bem como o acréscimo de atores, traz complexização à representação. $\mathrm{O}$ mapa pode crescer sempre que necessário.

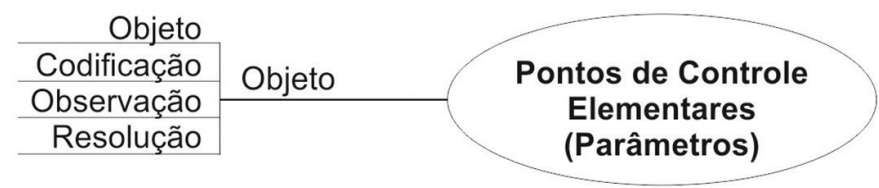

Figura 3. Mapeamento dos parâmetros da classe Objeto. Fonte: Elaborado pelos autores.

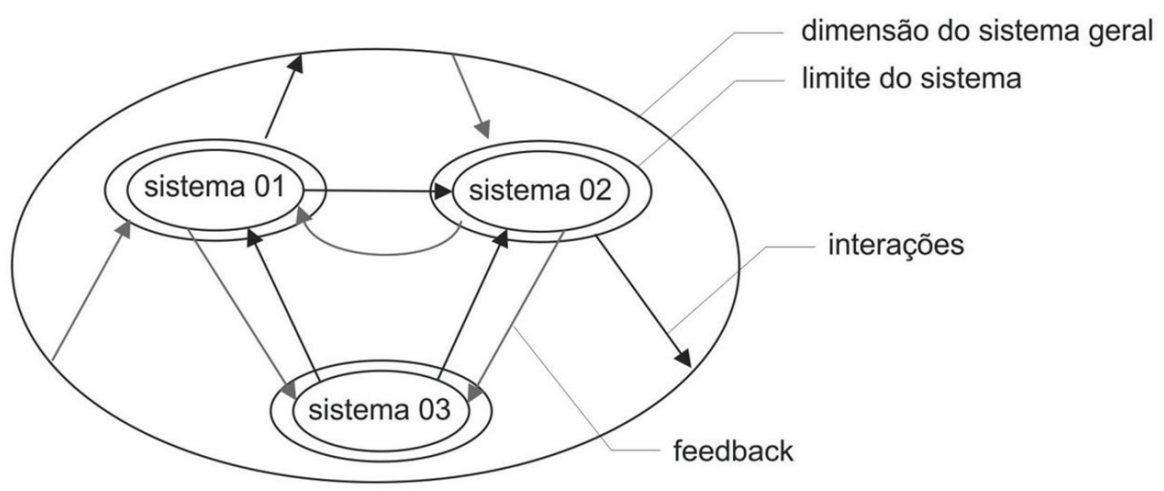

Figura 4.Diagrama de modelização para as relações Sistema-Sistema. Fonte: Elaborado pelos autores. 


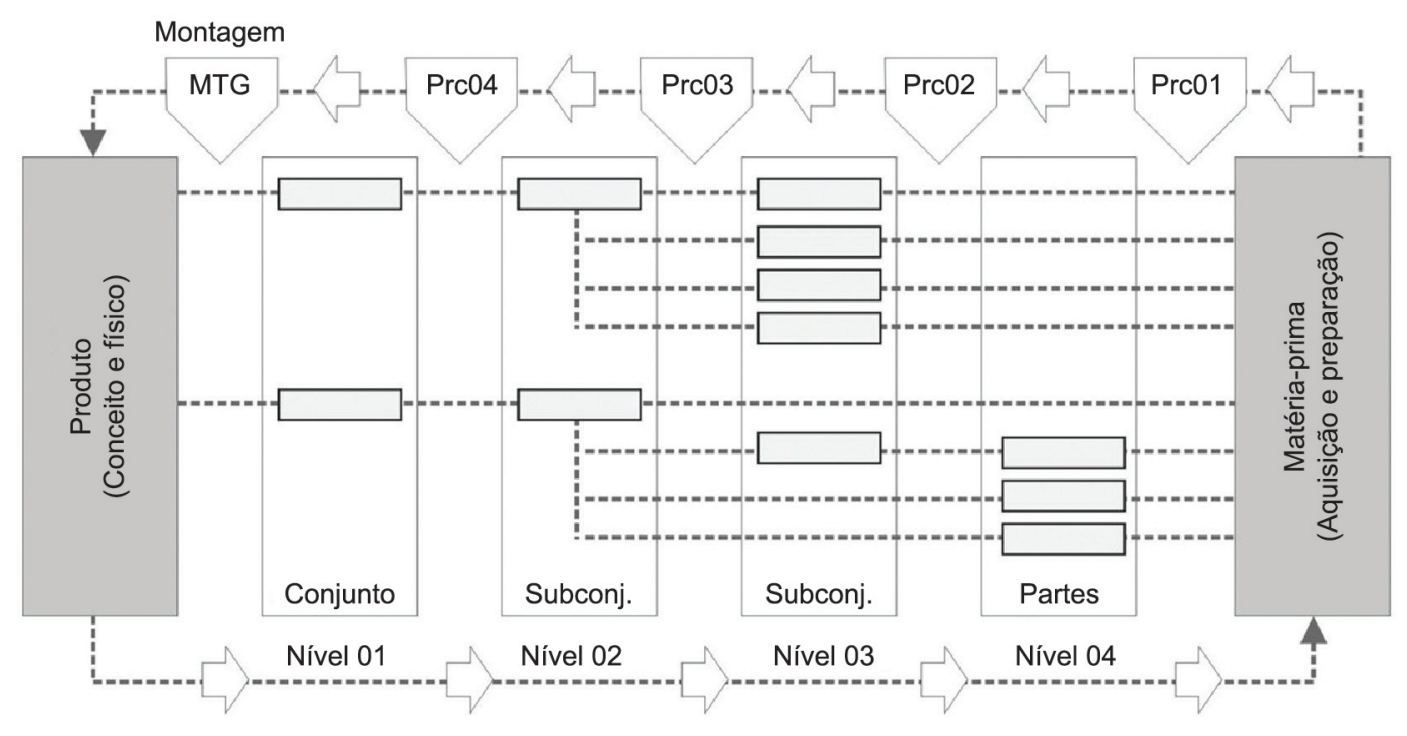

Linhas de relacionamento

Figura 5. Diagrama de Estruturação em Níveis. Fonte: Elaborado pelos autores.

\subsection{O diagrama para as relações Objetos-Transformações}

É um modelo de diagrama (Figura 5) que cuida do mapeamento das relações do sistema produtivo propriamente dito, ao representar as interações arborescentes, ou seja, sequências consecutivas e/ou concorrentes de atividades ou processos, típicas deste ciclo. Apresenta as características do aumento da resolução de modelagem e da necessidade da estruturação prévia do objeto a ser modelado.

Para esta estruturação, o diagrama conjuga mapa e fluxograma com o objetivo da representação de uma hierarquização em níveis, na qual o controle da transição do "projeto de um produto" para a "fabricação de um produto" seja válida e eficiente. Esta metodologia estabelece que seis níveis de estruturação, propiciam uma resolução de modelagem eficiente. Observa-se no diagrama que cada linha de relacionamento é potencial localização de ponto de controle. O fluxo inferior representa os processos da "desconstrução" do conceito em partes menores de informação até obtenção da representação do objeto elementar (a matéria-prima). É típico da atuação do parâmetro Informação. No fluxo superior, temos a "construção" do produto desde seu objeto físico elementar (a matéria-prima) até o produto acabado. É o fluxo típico das atuações dos parâmetros Processos e Eventos.

\subsection{O diagrama de casos de uso (UML)}

É um modelo de diagrama (Figura 6) que mostra a descrição de uma funcionalidade e o comportamento do sistema, como o usuário percebe e está diretamente

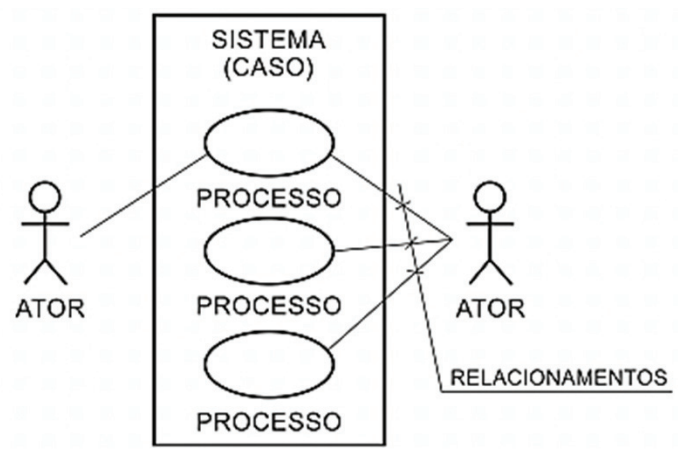

Figura6.DiagramadeCasodeUso. Fonte:OBJECT...(2005). Traduzido pelos autores.

relacionado aos atores. Ele representa um número de atores internos e externos e seus relacionamentos para cada uso específico do sistema. É uma unidade discreta da interação entre um usuário (humano ou máquina) e o sistema, descrevendo sequências de eventos para um ator que usa o sistema conseguir completar um processo (COCKBURN, 2005; ERIKSSON et al., 2004; OBJECT..., 2005).

A importância da especialização de ator é evidente.

\subsection{Diagramas de atividades (UML)}

É um modelo de diagrama (Figura 7) que representa um fluxo de ações e atividades realizadas em um fluxo geral de processos (workflow), podendo também especificar mensagens e transformações de objetos. Ele representa os fluxos conduzidos por processamentos, possibilitando o controle da transição de uma atividade para outra (WILSON FILHO, 2000; ERIKSSON et al., 2004; OBJECT..., 2005). 
Os diagramas de atividade são importantes para a modelagem de aspectos dinâmicos de um sistema.

\section{Exemplo de aplicação: o modelo conceitual para gerenciamento do desenvolvimento de um absorvedor sonoro}

Como exemplo de aplicação da metodologia, serão apresentados diagramas da modelagem conceitual do desenvolvimento de um produto. Não abrangem a totalidade da representação da complexidade exigida, entretanto apresentam número de pontos de controle significativos. O absorvedor sonoro em questão (Figura 8) é um componente arquitetônico para controle acústico desenvolvido pelo Projeto de
Pesquisa ECOPOLO - Sustentabilidade para o polo moveleiro do Vale do Jequitinhonha: Diversificação e valorização do uso de madeira de eucalipto por meio do design de componentes arquitetônicos e da certificação de produtos madeireiros (PEREIRA; SOUZA; PÊGO, 2008).

A proposta apresenta grande complexidade sistêmica, sendo necessária a adequação de: 1) conceitos eco-orientados para o produto; 2) conhecimentos sobre conforto ambiental; 3) critérios para atender às demandas de mercado; 4) critérios para incrementar a competitividade das empresas produtoras pelo comprometimento ambiental; 5) critérios de melhoria dos produtos pela adoção de metodologias de design; 6) critérios de implantação de processos de certificação; 7) critérios para conscientização da

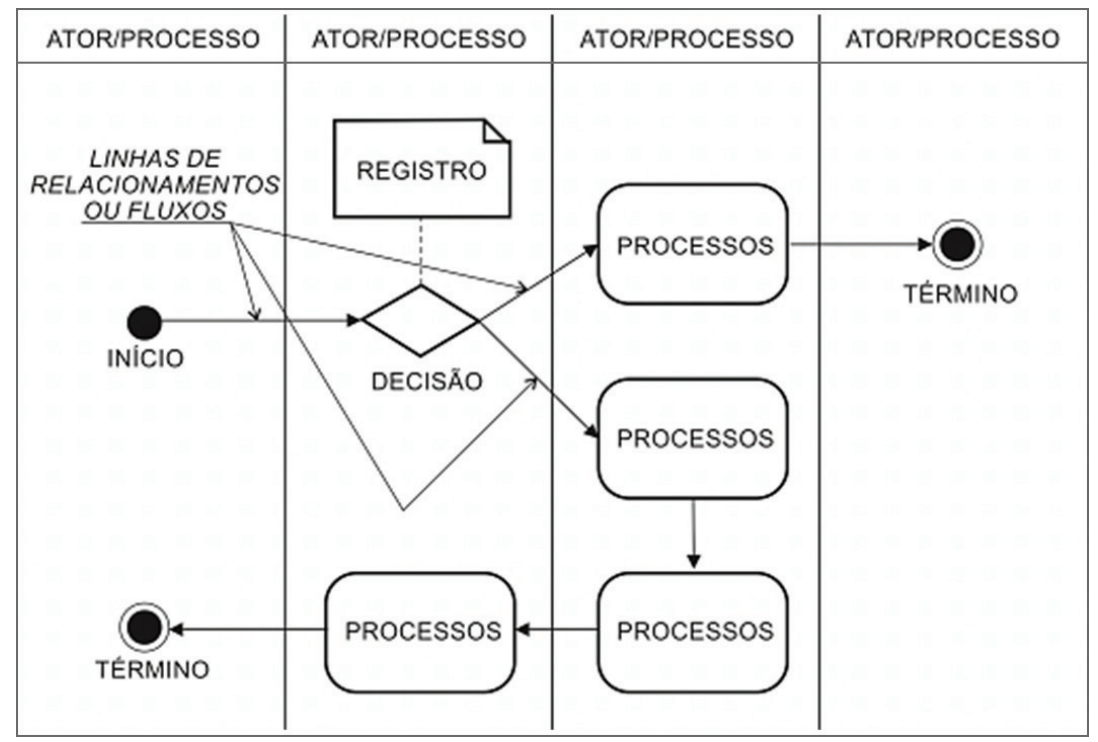

Figura 7. Diagrama de Atividades. Fonte: OBJECT... (2005). Traduzido pelos autores.

(a)

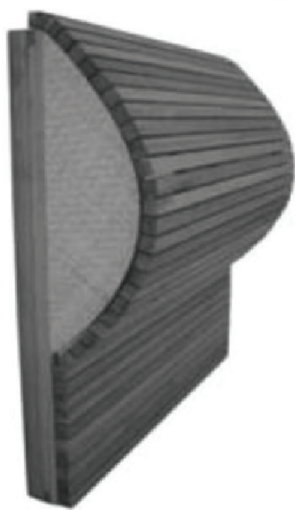

(b)

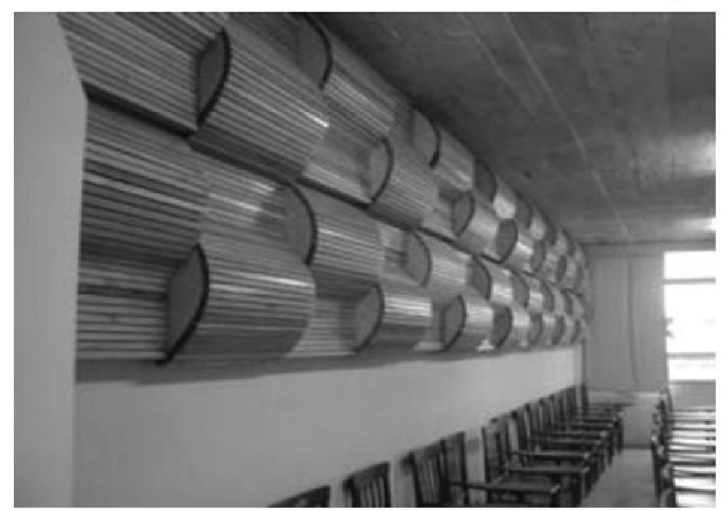

(c)

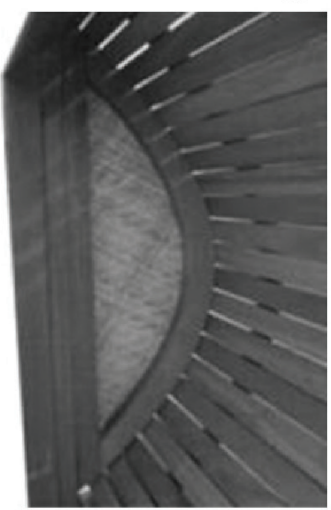

Figura 8. Absorvedor sonoro: a) unidade; b) detalhe interno da construção; c) instalação em uma sala da Escola de Arquitetura - UFMG. Fonte: Pereira, Souza e Pêgo, 2008. 
necessidade do controle da qualidade na estrutura produtiva atual; e 8) critérios de "projeto sustentável" para edificações (PEREIRA; SOUZA; PÊGO, 2008). A partir de cálculos sobre a quantidade de absorção sonora necessária para o conforto acústico de um dado ambiente especifica-se a quantidade de absorvedores sonoros que devem ser aplicados.

\subsection{Os sistemas envolvidos}

Focando no relacionamento entre os sistemas envolvidos, identificaram-se sete atores elementares: 1) o produtor do absorvedor sonoro; 2) o designer; 3) o cliente; 4) o fornecedor de madeira certificada; 5) o governo; 6) os órgãos normalizadores; e 7) os órgãos certificadores ambientais. O diagrama pode ser modelado conforme a Figura 9. Foi identificado um ponto de controle mínimo para cada evento de transição e relacionamento entre os sistemas:

A atuação dos atores e especialistas presentes nos sistemas foi representada por diagramas de Casos de Uso. Como exemplo, a Figura 10 representa as transações necessárias para se garantir uma compra válida de madeira certificada:

O negócio (compra de madeira certificada) será válido se todos os controles forem positivos.

\subsection{Conceito do produto e o sistema produtivo}

Focando o desenvolvimento do produto, conceituou-se o absorvedor sonoro como constituído de módulo caixa com geometria que possibilita a formação de um colchão de ar que permite a absorção do som, proporcionando um ambiente agradável. A parte curva apresenta absorção total de 24 UMA (unidades de métricas de absorção). Cabe à parte reta uma absorção de 7 UMA. Arruelas de cortiça são previstas para evitar que os ressonadores vibrem com as ondas sonoras e criam espaços entre eles, responsáveis pela entrada do som. São utilizados para a montagem, corda de sisal para amarração, trançado de fibras de cambaúba (um tipo de bambu) e parafusos metálicos para a fixação (PEREIRA; SOUZA; PÊGO, 2008). A estruturação do produto pode ser representada pelo diagrama objeto-transformações conforme a Figura 11. Observa-se que a produção dos componentes não utilizou os quatro níveis definidos pelo estudo. Os pontos de controle aparecem em cada linha de transição e também nas mudanças de níveis da estruturação:

A informação é validada no fluxo inferior e os processos no fluxo superior. Para o controle da

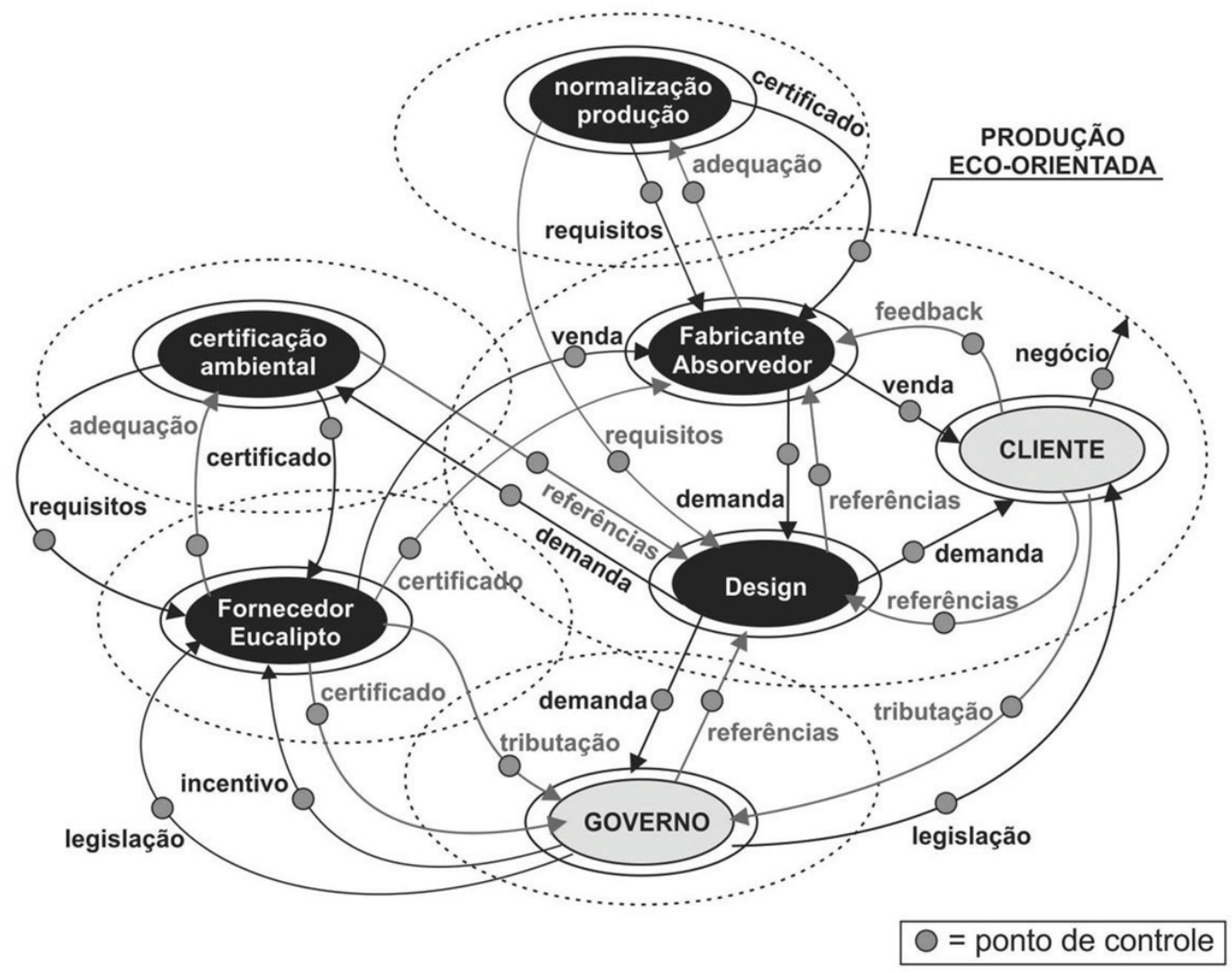

Figura 9. Modelagem dos eventos de transição e relacionamento dos sistemas envolvidos nos projeto do absorvedor sonoro. Representado em um diagrama sistema-sistema proposto pelo estudo. Fonte: Elaborado pelos autores. 


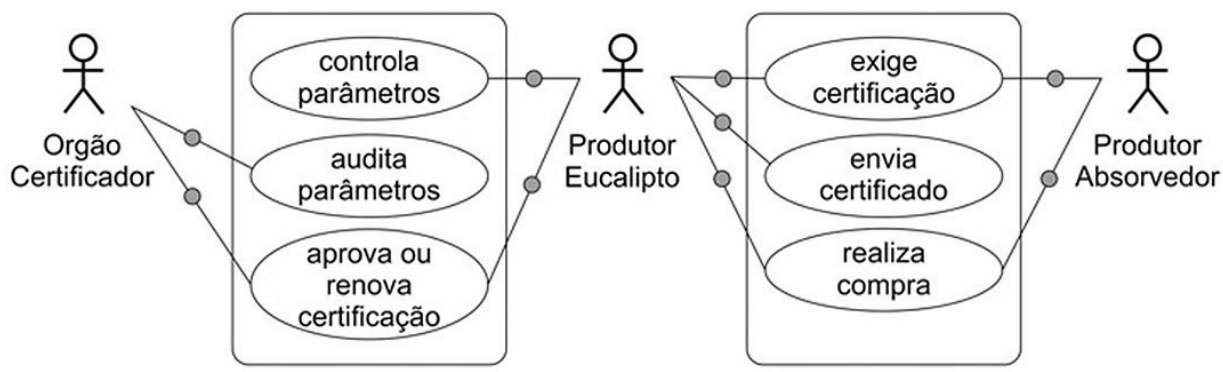

$\mathrm{O}=$ ponto de controle

Figura 10. Modelagem dos eventos de relacionamento entre um grupo de atores para a validação do processo de compra de madeira certificada. Fonte: Elaborado pelos autores.

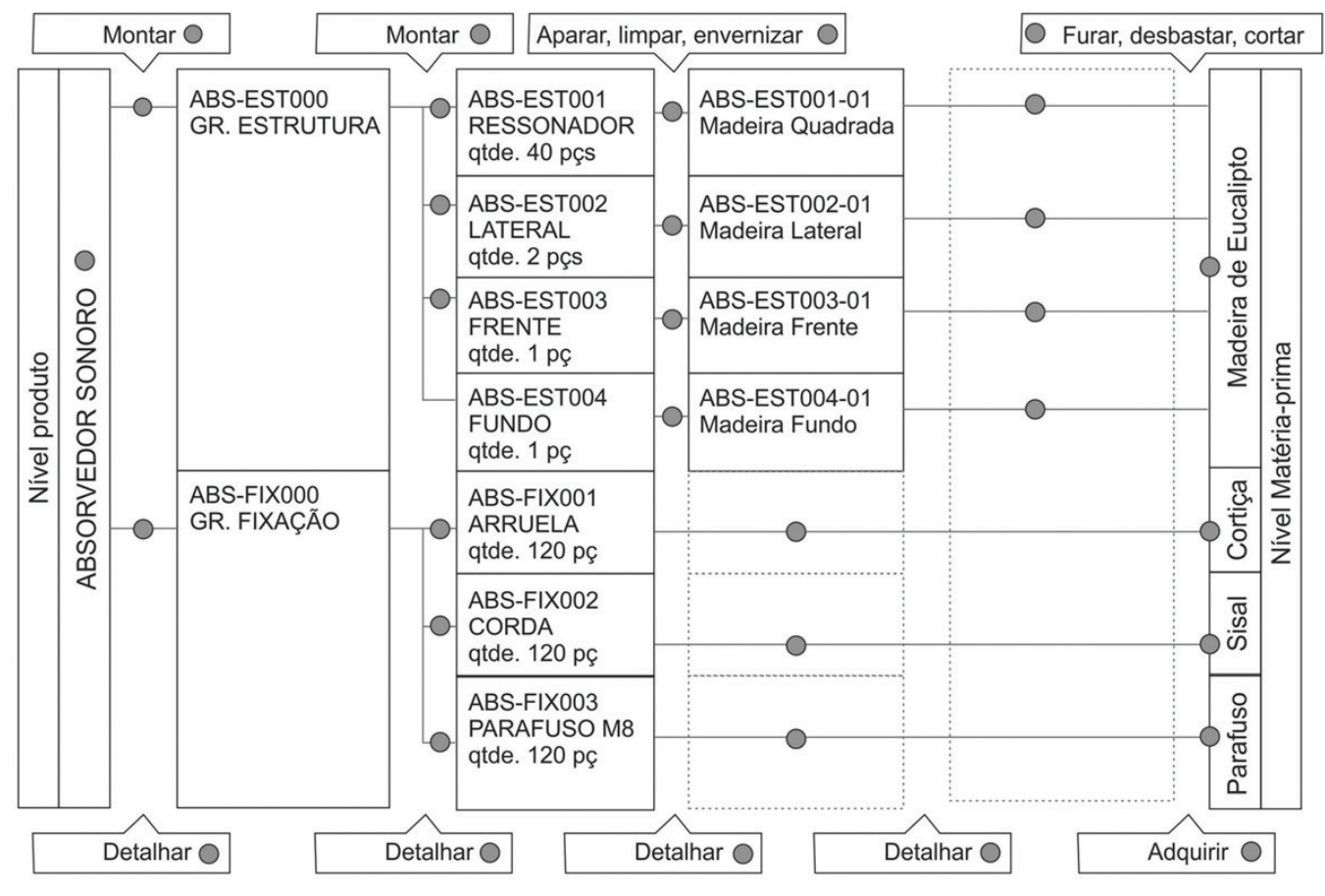

$=$ ponto de controle

Figura 11. Modelagem da estruturação do produto em níveis de organização e processos, representado em um Diagrama Objeto-transformação. Fonte: Elaborado pelos autores.

produção, utiliza-se o mapeamento do fluxo de produção, representado no diagrama de atividades UML na Figura 12.

Nesta representação, o controle dos processos (quantidade de pontos de controle) foi modelado com uma resolução maior, ou seja, com mais detalhamento dos objetos, suas relações, atores e processos. O trabalho de gestão é proposto após a análise de todos os mapeamentos. As práticas habituais das metodologias de gerenciamento podem ser adotadas, visto que a identificação de pontos de controle se mostra pertinente, pois atua em vários níveis de complexidade de todos os sistemas envolvidos.

\section{Desdobramentos e possibilidades de uso do estudo}

A proposta de se trabalhar com os diagramas de uma linguagem de modelagem unificada cria a possibilidade da geração de modelos conceituais que podem ser transformados em sistemas executáveis ou softwares de controle. Mas uma possibilidade interessante está na aproximação da modelagem dos diagramas com os modelos conceituais das interfaces 


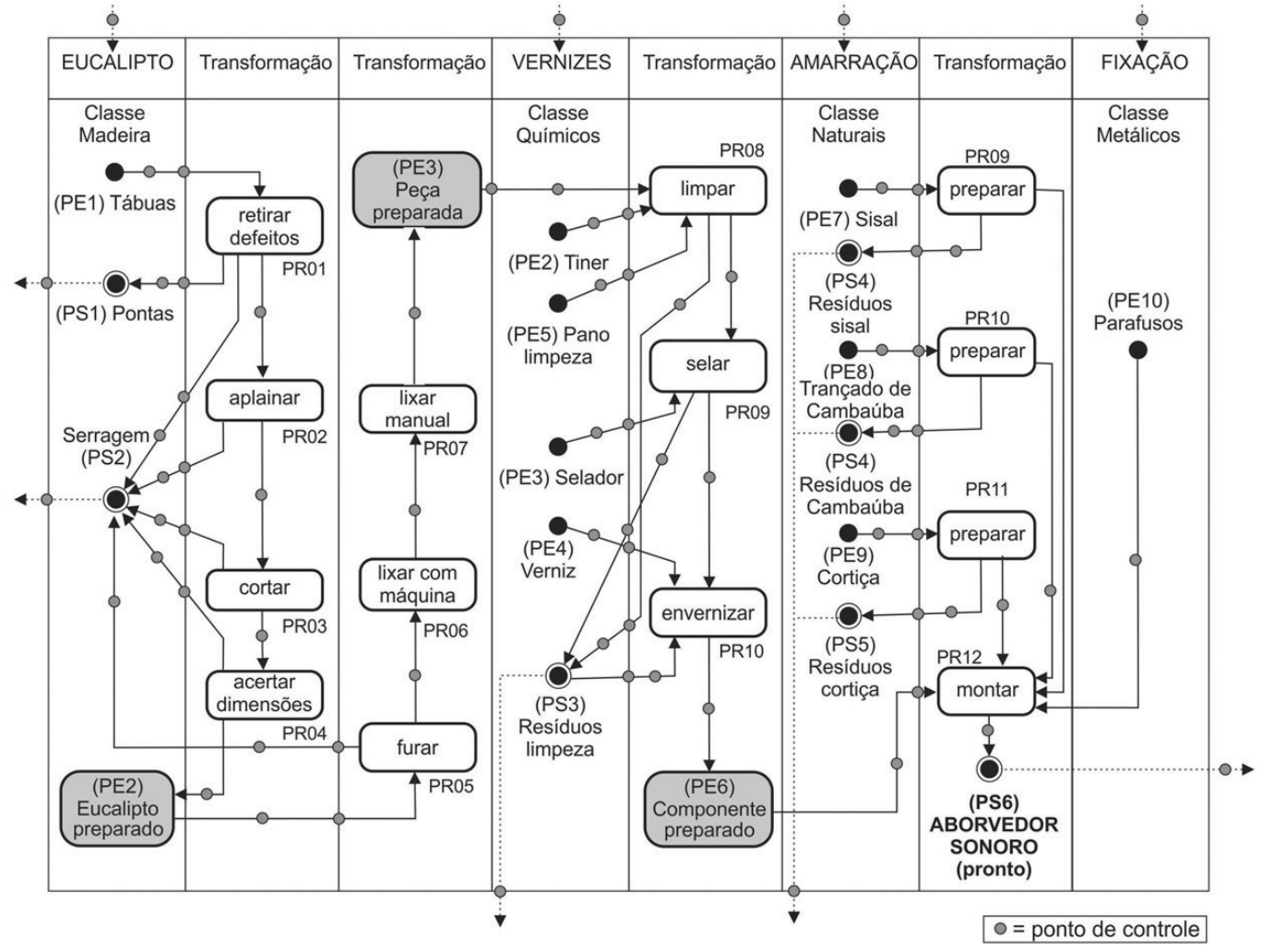

Figura 12. Modelagem dos fluxos produtivos para absorvedor sonoro, representado em um diagrama de Atividade - UML. Fonte: Elaborado pelos autores.

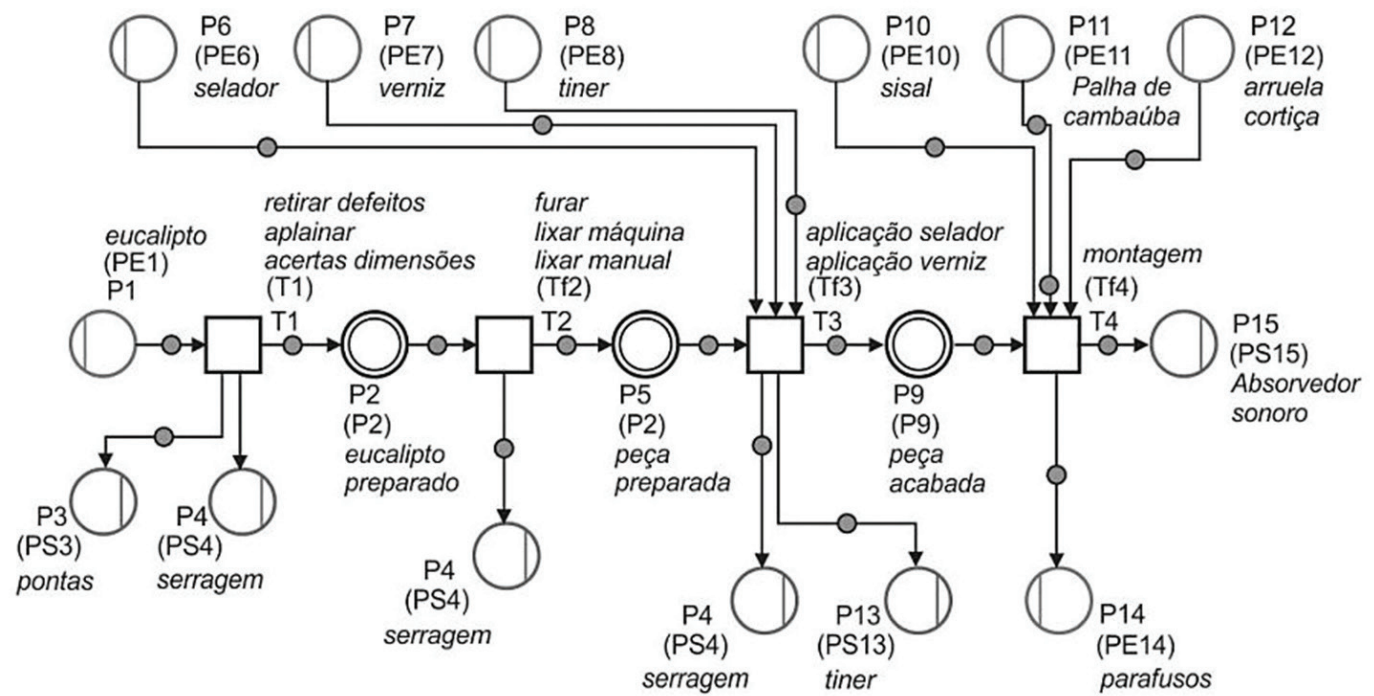

Figura 13. Modelagem do sistema produtivo do absorvedor sonoro na interface do software de ACV Umberto, modelados pelos autores.

com o usuário dos novos softwares de Análise do Ciclo de Vida. A ACV atua nos cálculos de balanço de massa e de energia dos sistemas e é método de avaliação que considera os aspectos ambientais em todas as diferentes fases que compõem a vida de um produto (CHEHEBE, 1997). A ACV estuda os aspectos ambientais e os impactos potenciais ao longo da vida de um produto desde a aquisição da matériaprima, passando por produção, uso e disposição (ASSOCIAÇÃO..., 2009). As normas avaliam o impacto da utilização-liberação de materiais e gastos energéticos, visando identificação da possibilidade 
de implementações de melhorias ambientais, além de aproveitar os parâmetros obtidos e objetivados para o desenvolvimento de novos modelos de gestão. $\mathrm{O}$ mercado dispõe atualmente de diversos softwares de ACV. Um exemplo pertinente ao estudo é o software Umberto, produzido na Alemanha pela Ifu Hamburg $\mathrm{GmbH}$. Este chama a atenção por possuir interface para geração conceitual do sistema que será analisado. É definido como software de análise de fluxo de materiais e cálculo de inventários (IFU..., 2011). Por tratar os fluxos, possibilita um mapeamento entre os atores (partes atuantes), componentes do sistema, evidenciando suas relações (rede de trabalho) e mudanças de estados (físicos, lógicos e logísticos). Também possibilita a criação de cenários a partir de um modelo original gerado para um determinado sistema. Caso se quisesse calcular o impacto ambiental para a produção do absorvedor sonoro, seria necessário modelar seu sistema produtivo na interface do software que apresentaria o diagrama da Figura 13:

Uma comparação com o diagrama de atividade apresentado anteriormente na Figura 12 demonstra similaridades para as linhas de fluxo, para os entrantes, efluentes, estados e transições, sendo totalmente compatíveis as representações de complexidade em ambos os diagramas.

\section{Conclusões}

A simplificação impõe disjuntar e reduzir e a complexidade ordena juntar e distinguir (LE MOIGNE, 1995). Com este estudo, buscou-se uma forma alternativa de entender a complexidade envolvida no desenvolvimento de um produto, além de representá-la de forma inteligível. Porém, ficou claro que somente com a junção de: 1) formas com as quais o ator que decide se relaciona com os outros especialistas; 2) formas com que identificamos pontos que validam um conceito; 3) identificação da informação que deva ser passada; e 4) forma com que se representa tudo isto, é possível trabalhar com a complexidade sem reducionismos.

$\mathrm{O}$ estudo mostrou que a modelagem da complexidade é um trabalho cíclico de juntar e distinguir, em inúmeros níveis. Isto até o momento no qual o ator decide se esta complexização do sistema que desenvolve atingiu sua expectativa. Deixou evidente a importância do papel dos atores envolvidos no desenvolvimento e do domínio de suas especializações, pois são eles os responsáveis pela utilização correta da informação exigida nos eventos do ciclo de vida. Às vezes, durante a observação do sistema ou produto (para o qual serão tomadas decisões), é o ator que define qual conteúdo de informação deverá ser distribuído e, então, se pode definir a localização do ponto de controle. Em outro caso, é evidente a necessidade de um ponto de controle em um determinado local do ciclo para que o conteúdo da informação seja definido.

A modelagem conceitual mostrou-se ferramenta importante para a representação e para o auxílio do desenvolvimento dos conceitos. Seu uso aponta para uma possibilidade de uso interdisciplinar interessante por propiciar mapeamentos de informação que auxiliam eficazmente a identificação das localizações no fluxo em que se deve controlar.

O que se observa é que o gerenciamento representa um ciclo no qual o conteúdo informacional, o ponto de controle, o evento, o conhecimento do processo e a especialização do ator possuem o mesmo peso. Um influencia a efetivação do outro e a falta de um deles representa risco ao controle do ciclo. Entretanto pode-se afirmar que é possível tratar o ciclo de vida de produto de maneira sistêmica, sem o perigo de uma redução em nível crítico da complexidade, utilizando modelagem conceitual. $\mathrm{O}$ desenvolvimento conjunto do conteúdo informacional propicia maior organização, produtividade, conhecimento do produto e, a partir disto, pode-se tomar melhores decisões.

\section{Referências}

AMARO, J. J. V. Considerações sobre a crise ambiental a partir de Karl Marx e Niklas Luhmann. In: ENCONTRO NACIONAL DA ANPPAS, 4., 2010, Florianópolis. Anais... Florianópolis, 2010.

ASSOCIAÇÃO BRASILEIRA DE NORMAS TÉCNICAS - ABNT. NBR ISO 14040:2009. Gestão ambiental Avaliação do Ciclo de Vida - Princípios e estrutura. Rio de Janeiro: ABNT, 2009.

BERTALANFFY, L. Teoria geral dos sistemas. Rio de Janeiro: Vozes, 2008.

CARDOSO, C. UML na prática: do problema ao sistema. Rio de Janeiro: Ciência Moderna, 2003.

CHEHEBE, J. R. B. Análise do ciclo de vida dos produtos Rio de Janeiro: Qualitymark, 1997.

CHENG, L.; LEONEL FILHO, D. R. M. Gestão de Desenvolvimento de Produtos (GDP) e o Método de Desdobramento da Função de Qualidade (QFD). Belo Horizonte: UFMG, 2006.

CNH GLOBAL NV. Global product development: overview and basics training session. USA: $\mathrm{CNH}, 2000$.

COCKBURN, A. Escrevendo casos de uso eficazes: um guia prático para desenvolvedores de software. Porto Alegre: Bookman, 2005.

COUGO, P. S. Modelagem conceitual e projeto de banco de dados. Rio de Janeiro: Campus, 1997.

ERIKSSON, H. et al. UML 2.0 Toolkit. 2nd ed. Indianapolis: Wiley, 2004.

GOMES, L. G. O. Design de informação e a modelagem conceitual nos softwares de ACV: estudo de caso Umberto. In: CONGRESO LATINOAMERICANO DE ENSEÑANZA DE DISEÑO, COMISIÓN SUSTENTABILIDAD Y ECODISEÑO EM LA ENSEÑANZA - MATERIALES E TECNOLOGÍAS SUSTENTABLES, 2., 2011, Buenos Aires. Anales... Buenos Aires, 2011. 
GOMES, L. G. Codificação e nomenclatura inteligentes: fundamentos para utilização e implantação do design da informação. In: CONGRESSO BRASILEIRO DE PESQUISA E DESENVOLVIMENTO EM DESIGN, 8., 2008, São Paulo. Anais... São Paulo, 2006.

IFU HAMBURG. Umberto 5.0: user manual. Hamburg, 2011.

LASTRES, H. M. M.; CASSIOLATO, J. E. (Org.). Glossário de arranjos e sistemas produtivos e inovativos locais. Rio de Janeiro: UERJ, 2003. Disponível em: <www. ie.ufrj.br/redesist>. Acesso em: 23 nov. 2012.

LE MOIGNE, J. La modélisation des systèmes complexes. 2. ed. Paris: Dunod, 1995.

MAXIMINIANO, A. C. A. Administração de projetos: como transformar idéias em resultados. 2. ed. São Paulo: Atlas, 2002.

MEADOWS, D. Places to intervence in a system. Hartland: Sustainability Institute, 1995.

MELO, A. C. Desenvolvendo aplicações com UML 2.0: do conceitual à implementação. Rio de Janeiro: Brasport, 2004.

MORIN, E.; LE MOIGNE, J. A inteligência da complexidade. Tradução de Nurimar Maria Falci. São Paulo: Petrópolis, 2000.

OBJECT MANAGEMENT GROUP. Infrastructure and standard document. Version 2.4.1. USA: OMG, 2005. Disponível em: <http://www.omg.org/spec/UML/2.4.1/ Infrastructure $>$.Acesso em: 05 ago 2012.
PEREIRA, A. F.; GOMES, L. Sistema de gestão integrada de desenvolvimento de produto para APL: Arranjo Produtivo Local. In: CONGRESSO BRASILEIRO DE PESQUISA E DESENVOLVIMENTO EM DESIGN, 7., 2006, Curitiba. Anais... Curitiba, 2006.

PEREIRA, A. F.; SOUZA, R. V. G.; PÊGO, K. Projeto ECOPOLO: design de componentes arquitetônicos visando a sustentabilidade nos edifícios e no Vale do Jequitinhonha. In: ENCONTRO NACIONAL DE TECNOLOGIA DO AMBIENTE CONSTRUÍDO, 2008, Fortaleza. Anais... Fortaleza, 2008.

PLOTKIN, J. Physical systems vs. conceptual systems. Amhers: University of Massachusetts, 2003. Disponível em: <http://www.physics.umass.edu/>. Acesso em: 03 nov. 2012.

SILVEIRA, A. Stage-Gate, aumente as chances de sucesso de seus produtos. São Paulo: 2006. Disponível em: <http://www.expleo.com.br>. Acesso em: 28 nov. 2012.

WILSON FILHO, P. P. Manual do engenheiro de software: módulo técnico. Rio de Janeiro: LTC, 2000.

WIENER, N. Cybernetics: or, control and communication in the animal and the machine. MIT Press, 1965. Disponível em: <http://books.google.com.br/books?id $=$ NnMuISyywAC\&printsec $=$ frontcover $\& d q=$ wiener $\&$ $\mathrm{hl}=\mathrm{pt}-\mathrm{BR} \& \mathrm{sa}=\mathrm{X} \& \mathrm{ei}=\mathrm{T} 1 \mathrm{v} 0 \mathrm{UIyHLsmS0QHlm} 4 \mathrm{CQBQ}$ \&ved=0CC8Q6AEwAA $>$. Acesso em: 16 maio 2012. 